\title{
Characterization of Hybrid Sol-gel Coatings Applied over Tinplate
}

\author{
D. Álvarez, ${ }^{*}$ A. Collazo, C. Pérez \\ ENCOMAT Group, E.E.I., University of Vigo, Campus Universitario, As Lagoas, \\ 36310 Vigo, Spain
}

\begin{abstract}
The present work studies the behavior of hybrid sol-gel films based on tetraethyl orthosilicate (TEOS) and vinyltrimethylsiloxane (VTMS) precursors applied over commercial tinplate. In previous works VTMS films obtained by the sol-gel technology were tested to verify their good corrosion performance over short immersion times in a citric acid/sodium citrate buffer solution. However, at longer exposure times the high porosity inherent to organic sol-gel films allows the aggressive media to reach the metallic substrate and start the corrosion process. In order to overcome these limitations, hybrid organic-inorganic sol-gel coatings were obtained using the dipcoating method. This work also studies the influence of the ageing time of the hybrid sol-gel in the formation of a uniform and continuous film.

The obtained layers were characterized using a Scanning Electron Microscopy (SEM) and mechanical profilometry. The corrosion performance in organic acid media was tested using polarization curves and Electrochemical Impedance Spectroscopy (EIS) with a $0.1 \mathrm{M}$ citric/citrate buffer solution as electrolyte.

The results obtained suggest that a minimum ageing time of 10 days is needed to obtain uniform films. The electrochemical measurements indicate a clear improvement on the barrier properties on the hybrid sol-gel compared to the organic ones. A major decrease in the impedance values after 24 hours of immersion indicates that it is necessary to improve the long-term barrier properties to achieve an industrial application.
\end{abstract}

Keywords: Sol-gel, tinplate, citric/citrate, EIS, equivalent circuit.

\section{Introduction}

Tinplate is the most widely-used raw material for manufacturing cans for the food packaging industry. Nowadays, the corrosion protection system includes a chromate pre-treatment and one or more organic lacquer layers to prevent

\footnotetext{
* Corresponding author. E-mail address: davidag@uvigo.es
} 
degradation of the tinplate in contact with the food. The chromate pre-treatment has three objectives: prevent the formation of sulfide staining during the sterilization process, improve the corrosion resistance and, finally, improve the adhesion of the subsequent organic coatings [1]. However, taking into account the toxicity of the waste water produced by the chromate pretreatment means, a more environmentally friendly alternative needs to be found. Several studies are exploring alternative pre-treatments using lanthanides, such as cerium or lanthanum, or transition metals oxides like titanium or vanadium oxides [2-5].

In recent years the epoxy-phenolic lining for the inside of the food cans have been subject to major controversy because of the precursors employed in their manufacture. Bisphenol-A (BPA) and its derivatives are widely employed as monomers in many different applications including the fabrication of epoxyphenolic lacquers. Several studies have demonstrated leaching of these compounds to the food or beverage from the container lining and also eventual absorption by the human body [6,7]. The effect of these compounds on human health is a matter of controversy between a part of the scientific community and the plastics industry. The effects of BPA as an estrogenic disruptor and tumor promoter are well documented in rodents [8,9]. Nowadays there are few epidemiological studies on the long-term effect in humans of exposure to low doses of BPA (reviewed in [6]). Nevertheless, there is greater sensitivity to the adverse effect of estrogenic disruptors in the embryonic and fetal stages, and some studies have revealed that BPA is capable of crossing the maternal-fetal placental barrier [10], which are issues that have increased concern among several national food safety organizations. In April 2008 Canadian authorities judged BPA and its derivatives to be hazardous to human life and health and banned polycarbonate baby bottles and the metal cans and lids of baby formula food. Following these restrictions, several countries restricted the use of BPA derivatives in the manufacture of materials intended to be in direct contact with food $[11,12]$. Finally, in France, BPA derivatives, including the epoxy-phenolic linings of metal cans, will be totally prohibited in the manufacturing of food containers [13].

These facts have motivated the canning industry's research effort into alternative coatings. Sol-gel coatings appear to be a promising alternative to traditional organic coatings and surface pre-treatments applied over different substrates $[14,15]$.

The sol-gel technique consists of the formation of an oxide network by hydrolysis and condensation reactions of metallic alkoxides. Organically modified alkoxysilanes were used as precursors to produce hybrid sol-gel coatings. These alkoxysilanes are characterized by the presence of at least one organic and non-hydrolizable group. The presence of the organic chains in the oxide network provides more flexibility to the coating and allows, when necessary, chemical compatibility with organic lacquers [16]. In the present work the precursors used were tetraethylortosilicate (TEOS) and vinyltrimethoxysilane (VTMS). The former is one of the most widely studied precursors in the synthesis of inorganic sol-gel thin layers [14], the latter is included in the list of precursors allowed in the manufacture of materials intended to be in direct 
contact with food (European Regulation $N^{\circ}$ 10/2011). VTMS films were tested by different authors and applied over different substrates such as aluminum $[17,18]$ or stainless steel [19].

The corrosion environment in the food canning industry involves significant concentrations of organic acid. In this work we use a $0.1 \mathrm{M}$ citric acid/ sodium citrate buffer solution to simulate the food media [20]. Citric acid is present in fruit and vegetables and is commonly used as electrolyte in electrochemical corrosion studies of tinplate and lacquered tinplate [21].

\section{Experimental \\ Materials}

TEOS and VTMS precursors were mixed with 2-propanol with a volume ratio of $1: 1$. In order to adjust the $\mathrm{pH}, 5.6 \%$ in volume of $\mathrm{HNO}_{3}$ solution was added $(\mathrm{pH}=0.5)$. Both solutions were mechanically stirred for 60 minutes at room temperature. After this stage, both solutions were mixed with a volume ratio TEOS/VTMS of 3:1 and maintained with mechanical and ultrasonic stirring for 60 minutes using an ultrasonic processor VCX 130® (from Sonics). After the synthesis, different ageing times were studied ranging from 1 day to 12 days before deposition.

The substrate employed was commercial tinplate (EN 10202 -TH620-CA-STE2,8-311-0,17×750) with a tin coating of $2.8 \mathrm{~g} \cdot \mathrm{m}^{-2}$ on both sides and a chromate passivation treatment (code 311 defined in EN 10202:2001). The tinplate samples $(3 \times 5 \mathrm{~cm})$ were cleaned before the deposition using an ultrasonic bath in acetone, and rinsed afterwards, first with soapy water and finally with distilled water. This treatment does not modify the original surface finish. The sol-gel film was obtained by dip-coating at $180 \mathrm{~mm} / \mathrm{min}$ with a holding time of 120 seconds. The samples were cured at $155^{\circ} \mathrm{C}$ for 20 minutes.

\section{Experimental techniques}

Several experimental techniques were employed in order to characterize the coatings and the corrosion performance of the sol-gel layers. All experiments were conducted at room temperature. Mechanical profilometry was employed to estimate the thickness of the coatings and their surface roughness. The surface profiles were obtained using a Dektak ${ }^{\circledR} 150$ Surface Profiler from Veeco. In order to obtain SEM images and EDX spectra, we used an Electroscan JSM-54 model JEOL 5410 equipped with an energy dispersive X-ray detector Link ISIS 300.

The electrochemical response of the coated samples to organic acid media was studied by means of polarization curves and electrochemical impedance spectroscopy (EIS) carried out in an AUTOLAB® 30 Potentiostat from Ecochemie. The measurements were conducted using a three electrode arrangement at room temperature. The working electrode was a delimited area of $1.3 \mathrm{~cm}^{2}$ of the tinplate sample, while the counter electrode employed was a graphite sheet and a saturated calomel electrode was used as reference. The electrolyte employed for both techniques was a $0.1 \mathrm{M}$ citric acid/sodium citrate 
buffer solution. The EIS results obtained for samples coated with sol-gel aged 1 day (TV75-1D) and 11days (TV75-11D) are presented in this paper.

The polarization curves were from $-700 \mathrm{mV}$ to $500 \mathrm{mV}$ vs. SCE with a scan rate of $0.4 \mathrm{mV} / \mathrm{s}$. The EIS spectra were measured in a frequency range from $10^{5} \mathrm{~Hz}$ to $10^{-2} \mathrm{~Hz}$ with 7 points per decade and amplitude of $10 \mathrm{mV}$ in the sinusoidal perturbation.

\section{Results and discussion}

\section{Morphological characterization}

As Fig. 1 shows, there are important differences in the layer formation depending on the ageing time. The coating obtained from the sol-gel solution aged one day shows a whitish color whereas the coatings obtained with eleven or more days of ageing are transparent. The whitish appearance disappears progressively as we increase the ageing time.

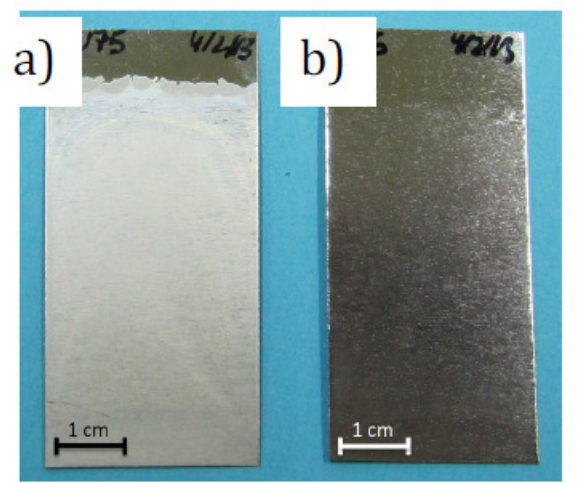

Figure 1. Images of the coatings obtained from sol-gel: a) aged 1 day; b) aged 11 days.

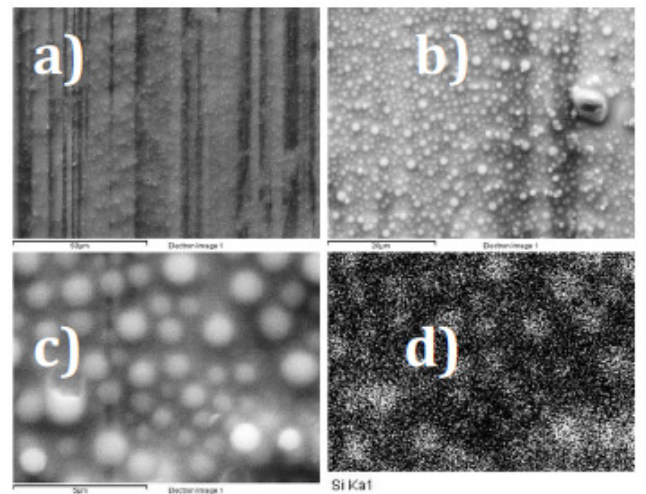

Figure 2. SEM images of the coatings obtained from sol-gel aged 1 day with different magnifications: a) 750x; b) 2000x; c) 10000x and d) Silicon EDX mapping of 10000x image.

The SEM images reveal substantial differences in the formation of the layers obtained with different ageing times. The formation of spheres with diameter around $1 \mu \mathrm{m}$ in the samples aged 1 day can be appreciated in Fig. 2. The generation of this kind of structure affects the distribution of the silicon oxide network over the tinplate substrate, which can be seen in the EDX mapping of silicon in Fig. 2. d). 
On the other hand, in Fig. 3 a continuous film of silicon oxides can be seen on the SEM images and EDX mappings obtained for the coatings with eleven days of ageing. The EDX mapping also shows the stripes of tin and iron-tin alloy typical of this kind of surface finish.
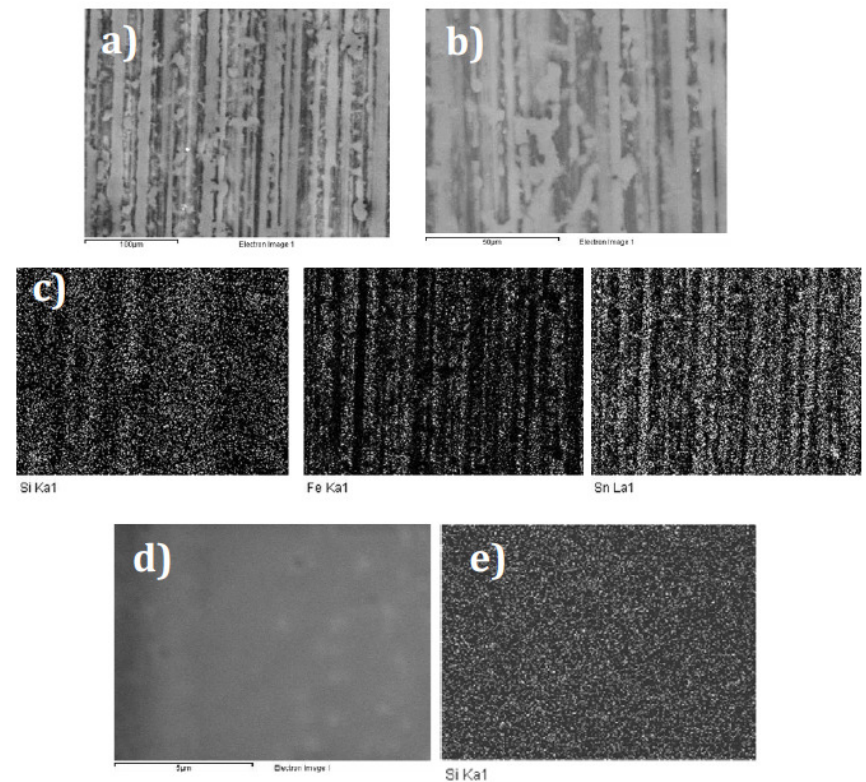

Figure 3. SEM Images of the coatings obtained from sol-gel aged 11 days with different magnifications: a) 350x, b) 750x, c) Si, Fe and Sn EDX mapping of the image obtained at 350x, d) SEM image at 10000x and e) Si EDX mapping at 10000x.

Using mechanical profilometry we can estimate the thickness of the layers obtained and study the amplitude parameters which define the surface roughness. $\mathrm{Ra}$ is defined as the average absolute deviation of the roughness irregularities from the mean line. Rt is defined as the vertical distance between the highest peak and the lowest valley. The values obtained are summarized in Table 1. It is noticeable that samples coated with sol-gel aged 11 days showed Ra values similar to those obtained for the bare tinplate. It seems that the coating follows the surface profile suggesting good wet ability. Rt values showed slightly lower values in this sample probably due a certain sol-gel accumulation in the valleys. On the other hand, the TV751D samples, even though they present a grainy appearance in the SEM images, showed lower Ra and Rt values over the coated surface. This could be explained by taking into account the lower viscosity of the sol-gel at short ageing times, which leads to greater accumulation of the sol-gel in the valleys, thus smoothing the surface roughness.

Table 1.Values obtained in the mechanical profilometry study.

\begin{tabular}{|c|c|c|c|}
\hline & Thickness $(\boldsymbol{\mu m})$ & $\mathbf{R a}(\boldsymbol{\mu m})$ & $\operatorname{Rt}(\boldsymbol{\mu m})$ \\
\hline TV75-1D & 1.045 & 0.060 & 0.640 \\
\hline TV75-11D & 1.380 & 0.230 & 1.36 \\
\hline Bare tinplate & -- & 0.220 & 1.90 \\
\hline
\end{tabular}


a)

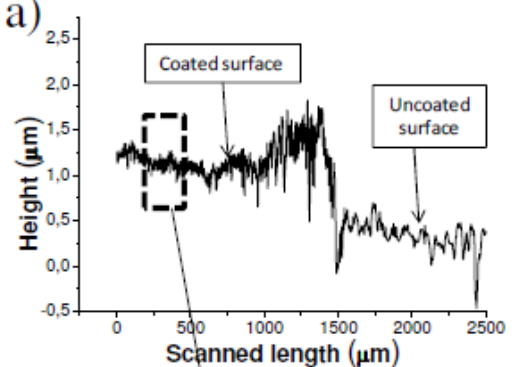

c)

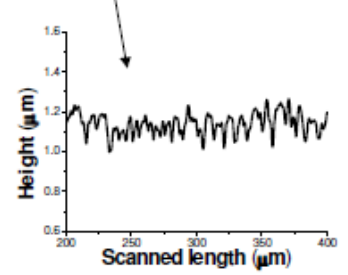

b)

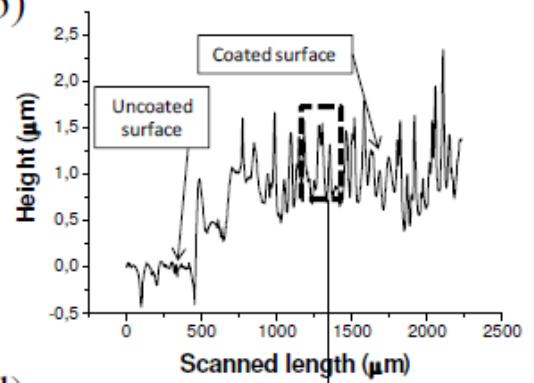

d)

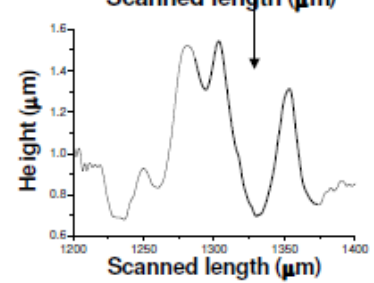

Figure 4. Surface profiles of the coated samples: a) TV751D, b) TV7511D, c) zoom of the highlighted area of surface profile a), and d) zoom of the highlighted area of surface profile b).

Fig. 4 shows two surface profiles of the coated samples with 1 and 11 days of ageing time. The total surface roughness of the coating is not affected by the formation of the sol-gel spheres due to their small diameter. The average diameter of the spheres is around $1 \mu \mathrm{m}$ (Fig. 2 c), clearly smaller than the broad peaks and valleys, which show widths around $20 \mu \mathrm{m}$. The surface profile obtained for the sample aged 1 day (Fig. 4 a) depicts this effect, showing higher roughness at lower scale. Nevertheless, when we analyze a higher scanned length the $\mathrm{Ra}$ values are clearly lower in the sample aged 1 day because the coating seems to follow the substrate topography better.

\section{Electrochemical characterization}

Potentiodynamic curves were recorded for coated samples with different sol-gel ageing times. The experiment was carried out after 30 minutes of immersion in the $0.1 \mathrm{M}$ citric/citrate buffer solution. As seen in Fig. 5, lower anodic current densities are registered as the ageing time increases, which indicates an improvement in the barrier properties [22]. Comparing the sample coated with sol-gel aged 1 day and the sample aged 11 days shows up a decrease of two orders of magnitude in the anodic current density.

The EIS measurements were taken after one hour of immersion in the citric/citrate buffer solution so that a stable potential could be reached. The Nyquist and Bode plots obtained are given in Fig. 6 and confirm the results obtained with the polarization curves. After 1 hour of immersion the best behavior was presented by the sample aged 11 days. The impedance value at low frequencies is two orders of magnitude higher in the TV75-11D sample than in the TV75-1D sample.

Two time constants can be clearly appreciated in the sample aged 11 days. However, in the sample aged 1 day the system presents a more complicated response with at least three time constants. To achieve a better understanding of 
the physical meaning of each time constant, we fit the experimental data to an equivalent circuit.

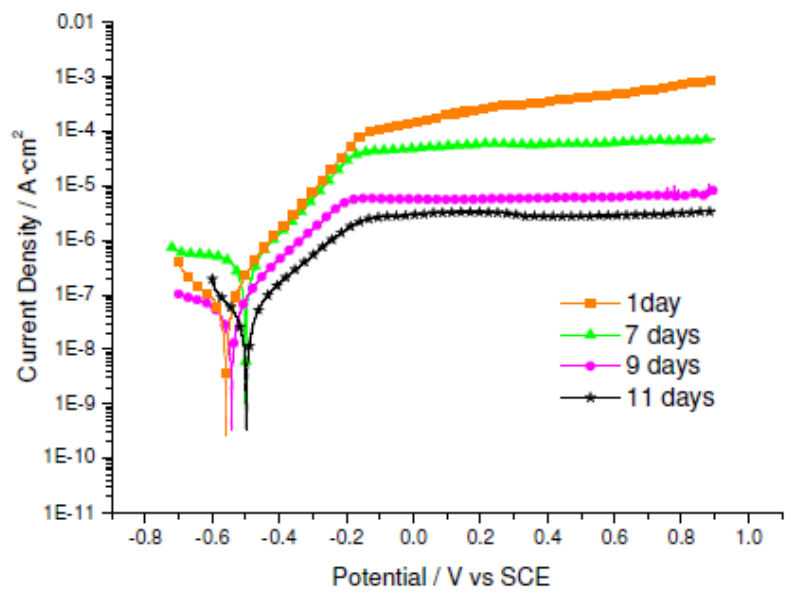

Figure 5. Polarization curves obtained for coated samples with different sol-gel ageing times after 30 minutes of immersion in $0.1 \mathrm{M}$ citric/citrate buffer solution.

In the sample aged 1 day, after 1 hour of immersion in the electrolyte we can appreciate three clear maximums in the phase angle in the Bode plot shown in Fig. 6. An equivalent circuit with three time constants in a parallel disposition gives the better fitting results (Fig. 7a). The time constant at high frequencies is related to the access of the electrolyte through the sol-gel coatings [23]. At lower frequencies we can detect the dielectric response of an oxide layer around 1 kilohertz [23] and the time constant related with the corrosion process around 1 hertz.
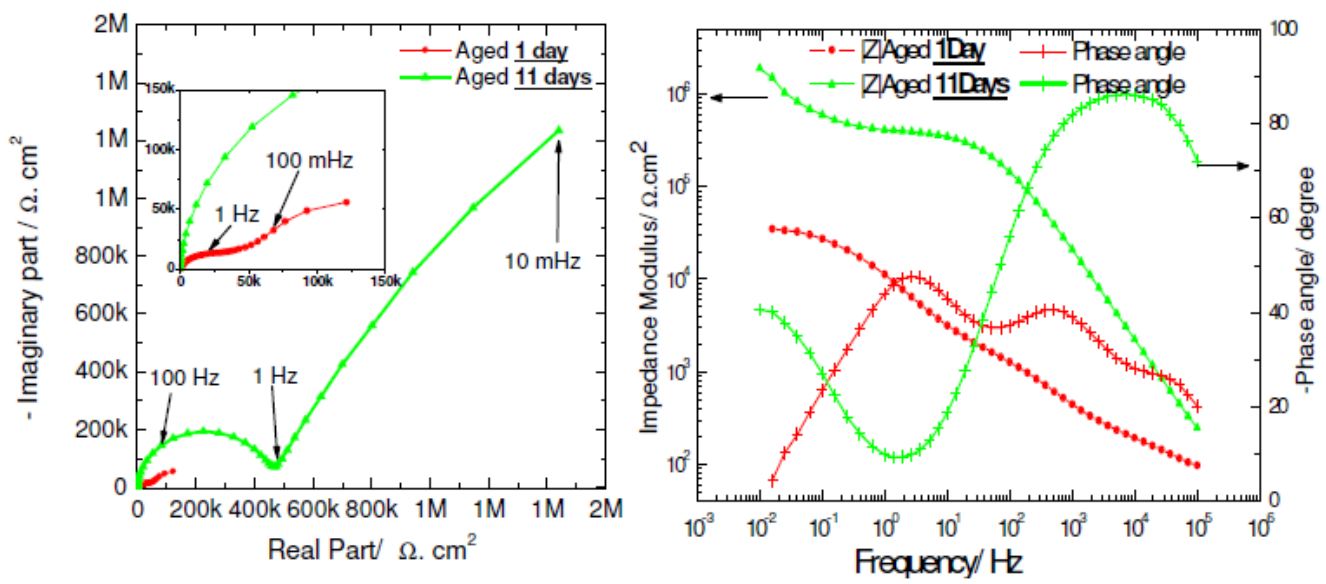

Figure 6. Nyquist and Bode plots obtained after 1 hour of immersion in $0.1 \mathrm{M}$ citric/citrate buffer solution.

On the other hand, for the sample aged 11 days, after 1 hour of immersion in the electrolyte, an equivalent circuit using two time constants in a parallel disposition gives the better fitting results. Likewise, the time constant at high frequencies is related to the barrier properties of the sol-gel coatings. The time constant related to the corrosion process appears in this case at lower frequencies and thus suggests lower corrosion rates. 

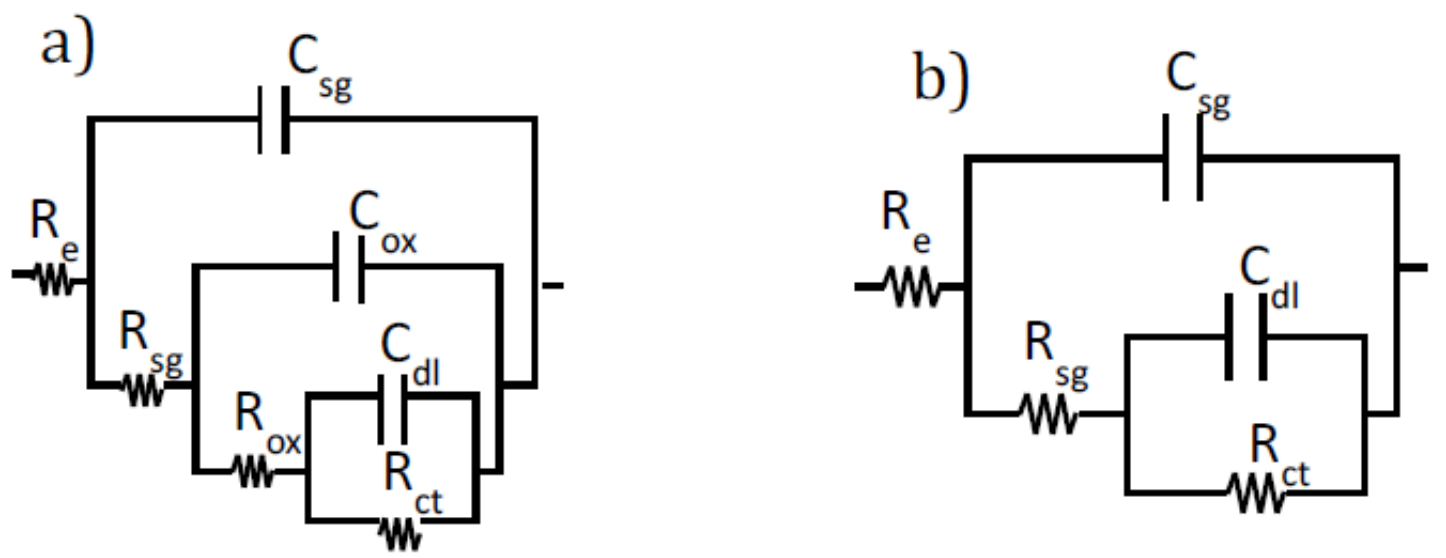

Figure 7. Equivalent circuits at short immersion times: a) for samples aged 1 day, and b) for samples aged 11days.

Table 2 gives the values obtained for the fitting after 1 hour of immersion in the electrolyte. $R_{e}$ is related to the electrolyte resistance. $R_{s g}$ and $C_{s g}$ are ascribed to the dielectric properties of the sol-gel coatings. The resistance is three orders of magnitude higher in the sample aged 11 days, indicating better barrier properties.

Table 2. Fitting values obtained after one hour of immersion in $0.1 \mathrm{M}$ citric/citrate buffer solution.

\begin{tabular}{|c|c|c|}
\hline \multicolumn{3}{|c|}{ Fitting values } \\
\hline & TV75-1D & TV75-11D \\
\hline $\operatorname{Re}$ & $67 \Omega . \mathrm{cm}^{2}$ & $68 \Omega . \mathrm{cm}^{2}$ \\
\hline Rsg & $229 \Omega . \mathrm{cm}^{2}$ & $427 \mathrm{k} \Omega . \mathrm{cm}^{2}$ \\
\hline Csg & $66 \mathrm{nF} . \mathrm{cm}^{-2}$ & $9 \mathrm{nF} . \mathrm{cm}^{-2}$ \\
\hline Rox & $2 \mathrm{k} \Omega . \mathrm{cm}^{2}$ & -- \\
\hline Cox & $0.5 \mu \mathrm{F} . \mathrm{cm}^{-2}$ & -- \\
\hline Rct & $36 \mathrm{k} \Omega . \mathrm{cm}^{2}$ & $18 \mathrm{M} \Omega . \mathrm{cm}^{2}$ \\
\hline Cdl & $16 \mu \mathrm{F} . \mathrm{cm}^{-2}$ & $17 \mu \mathrm{F} . \mathrm{cm}^{-2}$ \\
\hline
\end{tabular}

We can estimate the thickness of the sol-gel coating using Eq. 1 where $\mathrm{e}_{\mathrm{sg}}$ is the thickness of the sol-gel coating, $\varepsilon_{0}$ is the vacuum permittivity $\left(8.85 \times 10^{-14} \mathrm{~F} / \mathrm{cm}\right)$, $\varepsilon$ is the dielectric constant of the sol-gel layer and $\mathrm{C}_{\mathrm{sg}}$ the capacity associated with the dielectric properties of the coating. Assuming a dielectric constant of 10 for the water saturated sol-gel coating, this result is $1176 \mathrm{~nm}$ for the sample aged 11 days. This agrees well with the mechanical profilometry measurements. Nevertheless, in the sample aged 1 day the value obtained is only $134 \mathrm{~nm}$. The divergence between the result obtained and the profilometry measurements can be explained by taking into account the previous SEM images, which reveal the lack of homogeneity of samples like these.

$$
e_{3 g}=\frac{\varepsilon_{0} \times \varepsilon_{3 g}}{C_{g g}}
$$


The second time constant $\left(\mathrm{R}_{\mathrm{ox}}, \mathrm{C}_{\mathrm{ox}}\right)$ is related to the dielectric response of the oxides present in the passivation layer and the corrosion products. The presence of this time constant after only one hour suggests almost immediate access of the electrolyte to the substrate in the TV75-1D samples. Assuming a dielectric constant of $15 \mathrm{~F} / \mathrm{cm}$ [24] and using Eq. 2 we can make an estimation of the oxide layer thickness. The obtained value of $26 \mathrm{~nm}$ seems reasonable for an oxide layer.

$$
e_{o x}=\frac{\varepsilon_{0} \times \varepsilon_{o x}}{C_{o x}}
$$

Regarding the time constant related to the corrosion process, we can appreciate a value three orders of magnitude higher than the resistance of charge transference $\left(\mathrm{R}_{\mathrm{ct}}\right)$ in the sample aged 11 days, which means a slower corrosion process. Taking these results into account, it is clear than a minimum ageing time is necessary to obtain a homogeneous SG film.

Fig. 8 shows the evolution of the TV7511D samples over 24 hours. After this lapse of time a decrease in the impedance modulus of one order of magnitude was registered. Also a clear evolution to a three time constants system can be appreciated. The Nyquist plot depicted in Fig. 9 a) presents the typical shape of a diffusion process through a finite layer.

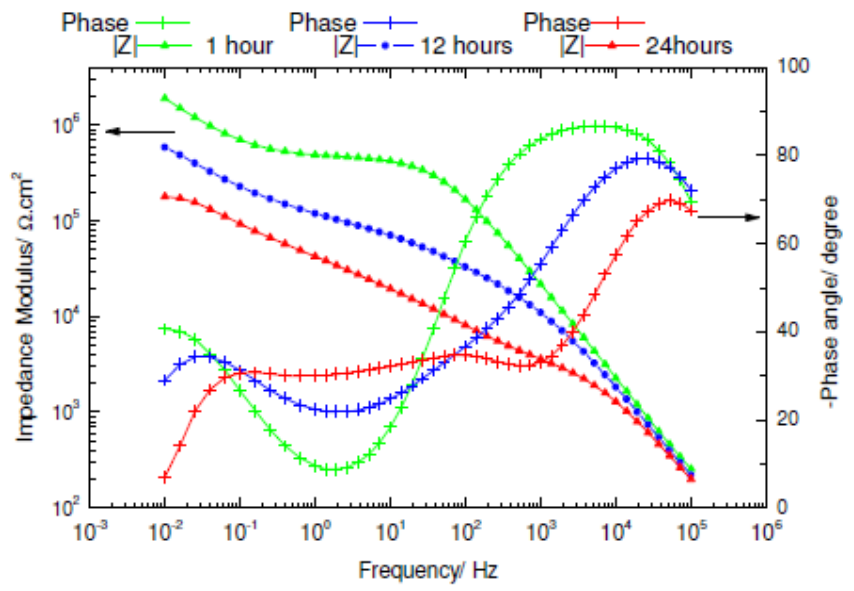

Figure 8. Bode plots obtained after 2, 12 and 24 hours of immersion in $0.1 \mathrm{M}$ citric/citrate buffer solution.

Fig. 9b) shows the equivalent circuit employed for the TV75-11D sample after 24 hours of immersion in the electrolyte. It presents two time constants in parallel disposition and a diffusion finite layer element $Z_{d}$ defined by Eq.3. However, it seems reasonable that there is the presence of a time constant related with the corrosion process at low frequencies, but the fitting results do not improve by adding another time constant. The effect of the time constant related to it could be overlapped by the dielectric response of the oxide layer. 


$$
Z_{d}=R_{d} \frac{\tanh \left(j w \tau_{d}\right)^{-\frac{1}{2}}}{\left(j w \tau_{d}\right)^{-\frac{1}{2}}}
$$

$R_{d}$ is the diffusion resistance and $\tau_{d}$ is the time constant associated with the diffusion process [25].

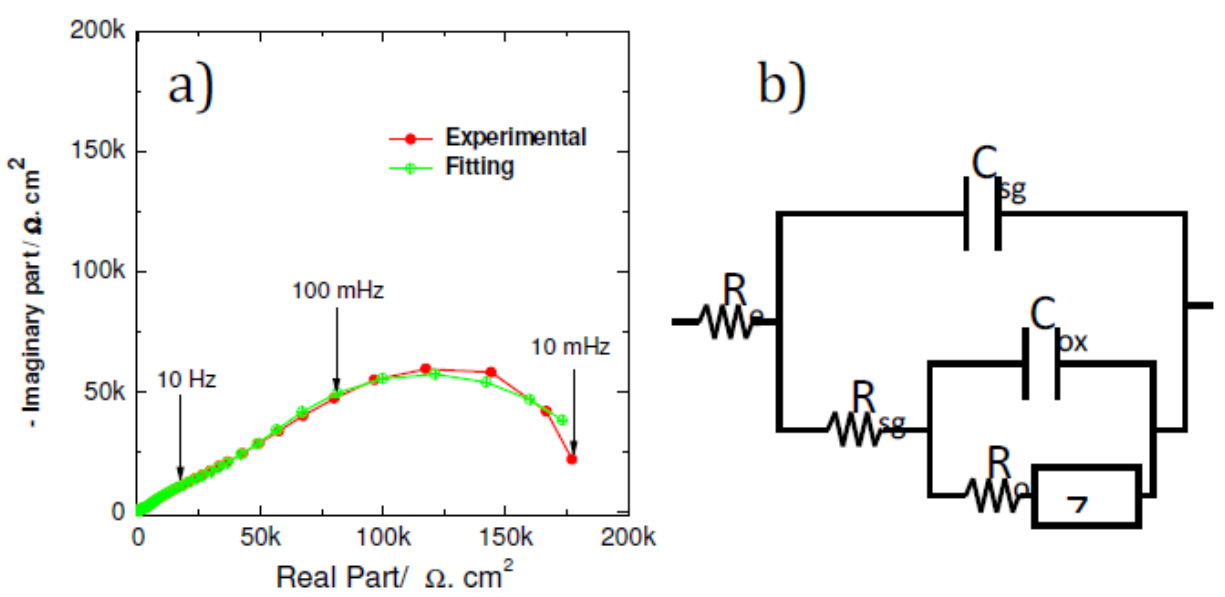

Figure 9. a) Measured and fitted Nyquist plots for TV7511D samples after $24 \mathrm{~h}$ of immersion in $0.1 \mathrm{M}$ citric acid/sodium citrate buffer solution, b) Equivalent circuit employed in the fitting of TV75-11D sample after 24 hours of immersion.

The fitting values obtained are summarized in Table 3. The rate of corrosion is limited, in this case, by the slow diffusion of oxygen through the sol-gel coating and the corrosion products. Using the fitting values and Eq. 4 we can estimate the thickness $(\delta)$ of this diffusion layer [25]. Assuming a diffusion coefficient (D) of the oxygen through the sol-gel coating of $1 \times 10^{-9} \mathrm{~cm}^{2} \cdot \mathrm{s}^{-1}$ [26] the value of $540 \mathrm{~nm}$ obtained is reasonable taking into account the thickness of this kind of coatings.

$$
\tau_{d}=\frac{\delta^{2}}{D}
$$

Table 3. Fitting values obtained after 24 hours of immersion in $0.1 \mathrm{M}$ citric/citrate buffer solution.

\begin{tabular}{|c|c|}
\hline \multicolumn{2}{|c|}{ Fitting values TV7511D } \\
\hline $\operatorname{Re}$ & $29.5 \Omega \cdot \mathrm{cm}^{2}$ \\
\hline $\operatorname{Rsg}$ & $2.1 \mathrm{k} \Omega \cdot \mathrm{cm}^{2}$ \\
\hline Csg & $13.8 \mathrm{nF} . \mathrm{cm}^{-2}$ \\
\hline $\operatorname{Rox}$ & $32.2 \mathrm{k} \Omega . \mathrm{cm}^{2}$ \\
\hline Cox & $1.2 \mu \mathrm{F} . \mathrm{cm}^{-2}$ \\
\hline $\mathrm{Rd}$ & $123 \mathrm{k} \Omega . \mathrm{cm}^{2}$ \\
\hline$\tau \mathrm{d}$ & $2.9 \mathrm{~s}$ \\
\hline
\end{tabular}




\section{Conclusions}

The results obtained by SEM and mechanical profilometry indicate that tinplate samples were coated with thin hybrid sol-gel layers. In all cases the thickness is around $1 \mu \mathrm{m}$. The ageing time strongly affects layer formation and, as a consequence, the corrosion performance.

Ageing times of eleven days or more are required to obtain homogeneous and transparent coatings. As far as the potentiodynamic curves and the EIS results are concerned, it can be observed that the layers obtained with these ageing times showed good corrosion performance at short immersion times in $0.1 \mathrm{M}$ citric acid/sodium citrate buffer solution. An equivalent circuit with two time constants in a parallel disposition is proposed to model the behavior of these coatings for short immersion times. However, after 24 hours of immersion the addition of a finite diffusion element to the equivalent circuit is needed due to the effect of oxygen diffusion through the sol-gel layer and the incipient corrosion products formed. The EIS study reveals a loss of barrier properties after 24 hours of immersion in the citric/citrate buffer solution.

The long-term barrier properties in layers like these must be improved if they are to be applied industrially.

\section{References}

1. Huang X, Li N, Wang H, et al. Thin Solid Films. 2008;516:1037-1043.

2. Mora N, Cano E, Polo JL, et al. Corros Sci. 2004;46:563-578.

3. Huang X, Li N. Appl Surf Sci. 2007;254:1463-1470.

4. Huang X, Li N. J. Alloys Compounds. 2008;465:317-323.

5. Zou Z, Li N, Li D, et al. J Alloys Compounds. 2011;509 :503-507.

6. Vandenberg LN, Hauser R, Marcus M, et al. Reprod Toxicol. 2007;24:139177.

7. Blunden S, Wallace T. Food Chem Toxicol. 2003;41:1651-1662.

8. Richter CA, Birnbaum LS, Farabollini F, et al. Reprod Toxicol. 2007;24:199-224.

9. Keri RA, Ho S, Hunt PA, et al. Reprod Toxicol. 2007;24:240-252.

10. Ikezuki Y, Tsutsumi O, Takai Y, et al. Human Reprod. 2002;17:2839-2841.

11. US Food and Drug Administration. 2010.

12. European Food Safety Authority. EFSA J. 2008;759:1-10.

13. LOI n ${ }^{\circ}$ 2012-1442. 1442 (2012).

14. Metroke TL, Parkhill RL, Knobbe ET. Prog Org Coat. 2001;41:233-238.

15. Wang D, Bierwagen GP. Prog Org Coat. 2009;64:327-338.

16. Zhu D, van Ooij WJ. Corros Sci. 2003;45:2177-2197.

17. Zand BN, Mahdavian M. Electrochim Acta. 2007;52:6438-6442.

18. Hu J, Liu L, Zhang J, et al. Prog Org Coat. 2007;58:265-271.

19. Motalebi A, Nasr-Esfahani M, Ali R, et al. Prog Nat Sci: Mater Int. 2012;22:392-400.

20. Gouda VK, Rizkalla EN, Abd-El-Wahab S, et al. Corros Sci. 1981;21:1-15.

21. Bastidas JM, Cabañes JM, Catalá R. Prog Org Coat. 1997;30:9-14.

22. Almeida E, Costa MR, Cristofaro N, et al. JCT Research. 2004;1:103-109. 
23. Zheludkevich ML, Serra R, Montemor MF, et al. Electrochim Acta. 2005;51:208-217.

24. Batzill M, Diebold U. Prog Surf Sci. 2005;79:47-154.

25. Scully JR. Corrosion. 2000;56:199-218.

26. Perez C, Collazo A, Izquierdo M, et al. Prog Org Coat. 1999;36:102-108. 